\title{
Track Billiards
}

\section{Leonid A. Bunimovich ${ }^{1}$, Gianluigi Del Magno ${ }^{2}$}

1 ABC Math Program and School of Mathematics, Georgia Institute of Technology, Atlanta, GA 30332, USA. E-mail: bunimovh@math.gatech.edu

2 Max Planck Institute for the Physics of Complex Systems, 01187 Dresden, Germany. E-mail: delmagno@mpipks-dresden.mpg.de

Received: 21 April 2008 / Accepted: 31 October 2008

Published online: 26 February 2009 - (C) The Author(s) 2009. This article is published with open access at Springerlink.com

\begin{abstract}
We study a class of planar billiards having the remarkable property that their phase space consists up to a set of zero measure of two invariant sets formed by orbits moving in opposite directions. The tables of these billiards are tubular neighborhoods of differentiable Jordan curves that are unions of finitely many segments and arcs of circles. We prove that under proper conditions on the segments and the arcs, the billiards considered have non-zero Lyapunov exponents almost everywhere. These results are then extended to a similar class of 3-dimensional billiards. Interestingly, we find that for some track billiards, the mechanism generating hyperbolicity is not the defocusing one, which requires every infinitesimal beam of parallel rays to defocus after every reflection off of the focusing boundary.
\end{abstract}

\section{Introduction}

There are rather few examples of hyperbolic billiards with several ergodic components, which are exactly described (for example, see [W2,B3,B-D1]). In this paper, we study a class of billiards whose phase space consists (up to a set of zero measure) of two invariant sets formed by orbits moving in opposite directions. The table of such a billiard is a tubular neighborhood of a differentiable Jordan curve $\gamma$ composed of finitely many straight segments and arcs of circles. A simple example of one of these tables is obtained by cutting out a smaller stadium from a stadium (Fig. 2(a)). Since these regions resemble track fields, our billiards will be called track billiards.

In this paper, we prove that all the Lyapunov exponents of a track billiard are nonzero almost everywhere provided that two conditions are satisfied: 1) the arcs of $\gamma$ are sufficiently long or the width of the cross section of the track is sufficiently large, and 2 ) the straight segments of $\gamma$ are sufficiently long. In addition, we show that a similar result remains valid for a class of 3-dimensional track billiards. There is no doubt that as a consequence of the hyperbolicity (non-zero Lyapunov exponents), each invariant 
set formed by orbits moving in one of the two possibile directions is ergodic. We will address this problem in a future paper.

Interestingly, we find that for some track billiards, the mechanism generating hyperbolicity is not the defocusing one. This mechanism requires that after every reflection from the focusing part of the billiard boundary, every narrow beam of parallel rays must pass through a conjugate point, and become divergent before the next collision with the curved part of the boundary. It follows from our results that there is a class of track billiards that are hyperbolic, but do not have this property.

It is worth mentioning that track billiards are related to billiards in tubular regions, which model certain electronic devices used in nanotechnology. Although, there are several works devoted to the study of the quantum properties of these billiards [E-S, G-J, C-D-F-K, V-P-R], not much attention has been dedicated to the study of their classical properties [H-P,P]. Our results, may help fill in this gap.

The paper is organized as follows. In Sect. 2, we review some basic facts concerning billiard systems, introduce tracks billiards, and state the main result of this paper. The last part of Sect. 2 contains some preliminary lemmas that are crucial for the proof of the hyperbolicity. In Sect. 3, we give the notions of focusing time and invariant cone field. Then, using a sort of generalized mirror formula for billiard trajectories crossing annular regions, we construct an eventually strictly invariant cone field for track billiards, whose existence implies hyperbolicity. Finally, in Sect. 4, the results obtained for 2-dimensional track billiards are extended to 3-dimensional track billiards.

\section{Track Billiards}

Let $Q$ be a bounded domain of $\mathbb{R}^{2}$ with piecewise differentiable boundary. The billiard in $Q$ is the dynamical system arising from the motion of a point-particle inside $Q$ obeying the following rules: the particle moves along straight lines at unit speed until it hits the boundary of $Q$, at that moment, the particle gets reflected so that the angle of reflection equals the angle of incidence.

2.1. Definitions. The domain $Q \subset \mathbb{R}^{2}$ considered in this paper is a tubular neighborhood of a differentiable Jordan curve $\gamma$ that is a finite union of segments and arcs of circles. Equivalently, we can say that $Q$ is a union of finitely many building blocks of two types: circular guides and straight guides. A circular guide is a region of an annulus with circles of radii $r_{1}>r_{2}>0$ contained inside a sector with central angle $0<\alpha<2 \pi$ (see Fig. 1(a)). A straight guide is simply a rectangle (see Fig. 1(b)). The circular and straight guides must all have the same transverse width in order to fit together and form a domain $Q$. Furthermore, we will always assume that any two circular guides of $Q$ do

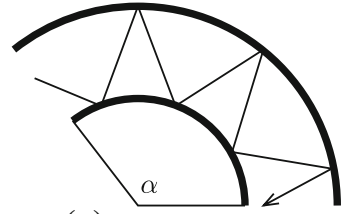

(a) Circular guide

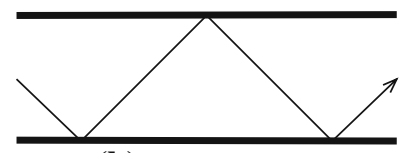

(b) Straight guide

Fig. 1. The two types of guides considered in this paper 

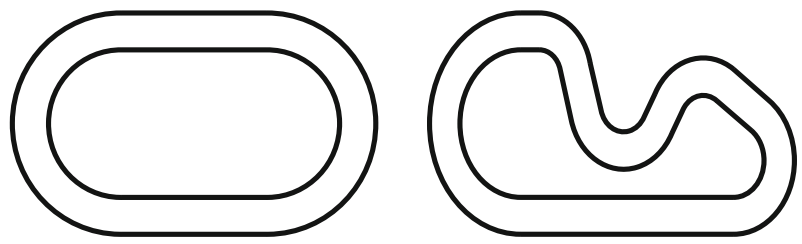

Fig. 2. Two examples of tracks

not intersect, i.e., they are separated by a straight guide. We call $Q$ a track, because its shape resembles that of a track field. Two examples of tracks are depicted in Fig. 2.

For our purposes, the dynamics of a track billiard can be conveniently described by a discrete transformation called the billiard map, which is defined as follows. Let $M$ be the set of all vectors $(q, v) \in T_{1} \mathbb{R}^{2}$ such that $q \in \partial Q$ and $\langle v, n(q)\rangle \geq 0$, where $n(q)$ is the normal vector to $\partial Q$ at $q$ pointing inside $Q$. Here $\langle\cdot, \cdot\rangle$ is the standard dot product of $\mathbb{R}^{2}$. The set $M$ is easily seen to be a smooth manifold with boundary. Let $\pi: M \rightarrow Q$ be the canonical projection defined by $\pi(q, v)=q$ for $(q, v) \in M$. If we view $q$ and $v$ as the position and the velocity of the particle after a collision with $\partial Q$, then $M$ represents the collection of all possible post-collision states (collisions, for short) of the particle with $\partial Q$.

Fix an orientation of the boundary $\partial Q$. A set of local coordinates for $M$ is given by $M \ni x \mapsto(s(x), \theta(x))$, where $s$ is the arclength parameter along the oriented boundary $\partial Q$, and $0 \leq \theta \leq \pi$ is the angle that the velocity of the particle forms with the oriented tangent of $\partial Q$. To specify an element $x \in M$, we will use either the notation $x=(q, v)$ or $x=(s, \theta)$. We endow $M$ with the Riemannian metric $d s^{2}+d \theta^{2}$ and the probability measure $d \mu=(2|\partial Q|)^{-1} \sin \theta d s d \theta$, where $|\partial Q|$ is the length of $\partial Q$.

Denote by $\partial M$ the set of all vectors $(q, v) \in M$ such that $\langle v, n(q)\rangle=0$ or $q$ is the endpoint of a straight segment of $\partial Q$. Let int $M=M \backslash \partial M$. For technical reasons, we define $T$ only on collisions belonging to the smooth manifold (without boundary) int $M$. The billiard map $T$ : int $M \rightarrow M$ is the transformation given by $(q, v) \mapsto\left(q_{1}, v_{1}\right)$, where $(q, v)$ and $\left(q_{1}, v_{1}\right)$ are consecutive collisions of the particle. Let us denote by $S_{1}^{+}$ the union of $\partial M$ and the subset of int $M$, where $T$ is not differentiable. It is easy to see that $S_{1}^{+}=\partial M \cup T^{-1} \partial M$. From the general results of [K-S], it follows that $S_{1}^{+}$is a compact set consisting of finitely many smooth compact curves that can intersect each other only at their endpoints. If we define $S_{1}^{-}=M \backslash T\left(M \backslash S_{1}^{+}\right)$, then $T$ is a diffeomorphism from $M \backslash S_{1}^{+}$to its image $M \backslash S_{1}^{-}$, and preserves the measure $\mu$ (see e.g. [C-F-S, K-S]).

The billiard dynamics is time-reversible. Indeed, the involution $\mathcal{J}: M \rightarrow M$ defined by $\mathcal{J}(s, \theta)=(s, \pi-\theta)$ for every $(s, \theta) \in M$ has the property that $\mathcal{J} \circ T=T^{-1} \circ \mathcal{J}$ everywhere on $M \backslash S_{1}^{+}$. Most of the time, we will use the notation $-A$ instead of $\mathcal{J} A$, where $A$ is a subset of $M$.

For every $n>1$, let us define $S_{n}^{+}=S_{1}^{+} \cup T^{-1} S_{1}^{+} \cup \cdots \cup T^{-n+1} S_{1}^{+}$and $S_{n}^{-}=S_{1}^{-} \cup T S_{1}^{-} \cup$ $\cdots \cup T^{n-1} S_{1}^{-}$. By the time-reversibility of the billiard dynamics, we have $S_{n}^{-}=-S_{n}^{+}$for every $n>0$. Let $S_{\infty}^{+}=\cup_{n>0} S_{n}^{+}$and $S_{\infty}^{-}=\cup_{n>0} S_{n}^{-}$. Then $\tilde{M}=M \backslash\left(S_{\infty}^{-} \cup S_{\infty}^{+}\right)$is the set where all iterates of $T$ are defined. Clearly, $\mu\left(S_{\infty}^{+}\right)=\mu\left(S_{\infty}^{-}\right)=0$ and $\mu(\tilde{M})=1$.

2.2. Unidirectionality. We say that a billiard in a track $Q$ has the unidirectionality property if every billiard trajectory that is not contained in a cross section of $Q$ moves through every cross section of $Q$ in the same direction. We will prove this property not only for 2-dimensional tracks but also for a certain class of tubular domains of $\mathbb{R}^{3}$. In fact, the 
unidirectionality of tracks will be derived from the undirectionality of 3-dimensional tubular domains. It would be easy to extend our proof to tubular domains in any dimension. We have not done that in order to keep the length of the proof within reasonable limits. We allow the cross section of the tubular domains to be an arbitrary convex compact subset of $\mathbb{R}^{2}$. In Sect. 4 , we will consider tubular domains of $\mathbb{R}^{3}$ with rectangular cross section. Because of the generality of the current setting, before proving the unidirectionality property, we provide a precise definition of a tubular domain in $\mathbb{R}^{3}$.

The 'skeleton' of the tubular neighborhood $\tilde{Q}$ is given by a regular Jordan curve $\varphi: S^{1} \rightarrow \mathbb{R}^{3}$ parametrized by the arclength $s$. We assume that $\varphi$ is piecewise $C^{2}$. This means precisely that there exist $a_{0}<b_{0}=a_{1}<b_{1}<\cdots<b_{n-1}=a_{n}<b_{n}=a_{0}$ such that $S^{1}=\cup_{1 \leq i \leq n}\left[a_{i}, b_{i}\right]$, and $\varphi$ is $C^{2}$ on each interval $\left[a_{i}, b_{i}\right]$. We also make the assumption that the curvature of $\varphi$ on each interval $\left[a_{i}, b_{i}\right]$ is either identically zero $\left(\varphi\left(\left[a_{i}, b_{i}\right]\right)\right.$ is a straight segment) or is never equal to zero. In the latter case, for every $s \in\left[a_{i}, b_{i}\right]$, let $\{T(s), N(s), B(s)\}$ be the Frenet frame of $\varphi$, where $T(s), N(s), B(s)$ are the tangent, normal and binormal vectors of $\varphi$ at $\varphi(s)$, respectively (see for instance [K1, Chap. 1]). In the former case, instead, we choose $N(s)$ and $B(s)$ to be some fixed vectors such that $\{T(s), N(s), B(s)\}$ forms an orthonormal basis of $\mathbb{R}^{3}$. The cross section of $\tilde{Q}$ is given by a compact convex subset $\Omega \subset \mathbb{R}^{2}$ whose boundary is a piecewise regular simple closed curve $\zeta: S^{1} \rightarrow \mathbb{R}^{2}$ with $\|\zeta(\alpha)\|>0$ for every $\alpha \in S^{1}=[0,2 \pi)$. The tubular neighborhood of $\varphi$ with cross section $\Omega$ is the domain $\tilde{Q}$ bounded by the surface $\psi(s, \alpha)=\varphi(s)+F(s) \zeta(\alpha)$ with $(s, \alpha) \in S^{1} \times S^{1}$, where $F(s)$ is the $3 \times 2$ matrix with column vectors given by $N(s)$ and $B(s)$. We suppose that $\tilde{Q}$ is not selfintersecting, namely, we require that the diameter of $\Omega$ is sufficiently small so that the map $\Phi: S^{1} \times \Omega \rightarrow \tilde{Q}$ given by $(s, p) \mapsto \varphi(s)+p$ is a diffeomorphism. In particular, we assume that $\max _{\alpha}\|\zeta(\alpha)\|<\left(\max _{s}|\kappa(s)|\right)^{-1}$, where $\kappa$ is the curvature of $\varphi$.

Proposition 1. Consider a tubular neighborhood $\tilde{Q}$ of $\mathbb{R}^{3}$, and assume that its cross section $\Omega$ is a circular disk or that each curve $\varphi\left(\left[a_{i}, b_{i}\right]\right)$ is planar. Then the billiard inside $\tilde{Q}$ has the unidirectionality property.

Proof. Let $s \in\left[a_{i}, b_{i}\right]$ and $\alpha \in[0,2 \pi)$. The vectors $\partial_{s} \psi=T(s)+F^{\prime}(s) \zeta(\alpha)$ and $\partial_{\alpha} \psi=F(s) \zeta^{\prime}(\alpha)$ span the tangent plane of $\partial \tilde{Q}$ at $\psi(s, \alpha)$. Consider the vector $\tilde{n}(s, \alpha)=$ $\partial_{s} \psi \wedge \partial_{\alpha} \psi$. We recall that the Frenet equations read as follows: $T^{\prime}(s)=\kappa(s) N(s)$, $N^{\prime}(s)=-\kappa(s) T(s)+\tau(s) N(s)$ and $B^{\prime}(s)=-\tau(s) N(s)$, where $\kappa$ and $\tau$ are the curvature and torsion of $\varphi$, respectively (see [Kl, Chap. 1]). A little lengthy but simple computation using the Frenet equations shows that

$$
\tilde{n}(s, \alpha)=\left(1-\zeta_{1}(\alpha) \kappa(s)\right) T(s) \wedge F(s) \zeta^{\prime}(\alpha)-\tau(s) F J \zeta(\alpha) \wedge F(s) \zeta^{\prime}(\alpha),
$$

where $\zeta_{1}(s)$ is the components of $\zeta(\alpha)$ along $N(s)$, and $J=\left(\begin{array}{cc}0 & 1 \\ -1 & 0\end{array}\right)$. Since $\zeta$ is piecewise regular, it follows that $\tilde{n}(s, \alpha) \neq 0$, which in turn guarantees that $\tilde{n}(s, \alpha)$ is parallel to the normal line to $\partial \tilde{Q}$ through $\psi(s, \alpha)$. It is now easy to obtain $\langle\tilde{n}(s, \alpha), T(s)\rangle=$ $-\tau(s)\left\langle\zeta(\alpha), \zeta^{\prime}(\alpha)\right\rangle$. By hypothesis, we have $\tau(s)=0$ ( $\varphi$ is planar) or $\left\langle\zeta(\alpha), \zeta^{\prime}(\alpha)\right\rangle=0$ ( $\zeta$ is a circle). Note that if $\varphi\left(\left[a_{i}, b_{i}\right]\right)$ is a straight segment, then $\tau(s)=0$. We can therefore conclude that $\langle\tilde{n}(s, \alpha), T(s)\rangle=0$, meaning that the plane orthogonal to $T(s)$ is orthogonal to the boundary of $\tilde{Q}$. It follows that the set $N=\{(q, v) \in M:\langle v, n(q)\rangle=$ $0\}$ is invariant. To obtain this conclusion, it is crucial that $\partial \tilde{Q}$ is not self-intersecting, otherwise the parametrization $\psi$ of $\partial \tilde{Q}$ ceases to be valid.

Consider now the billiard flow $t \mapsto(q(t), v(t))$ inside $\tilde{Q}$. Let $v_{*}(t)=\left\langle v(t), T_{*}(t)\right\rangle$, where $T_{*}(t)=T(s(t))$ and $s(t)$ is given by $(s(t), p(t))=\Phi^{-1}(q(t))$. Suppose that 
the particle motion is defined for $t \in(-\epsilon, \epsilon)$ with $\epsilon>0$, and that during such interval of time there is only one collision with $\partial \tilde{Q}$ at time $t=0$. We claim that $v_{*}$ is continuous on $(-\epsilon, \epsilon)$. First, note that $T_{*}$ is continuous on $(-\epsilon, \epsilon)$, and that $v$ is continuous on $(-\epsilon, 0) \cup(0, \epsilon)$. Thus to prove the claim, all we have to do is to show that $\lim _{t \rightarrow 0^{-}} v_{*}(t)$ and $\lim _{t \rightarrow 0^{+}} v_{*}(t)$ exist and coincide. The existence of these limits is obvious. Now, by the reflection law, we have $v\left(0^{+}\right)=v\left(0^{-}\right)+2\left\langle v\left(0^{-}\right), n(q)\right\rangle n(q)$. Since $\left.\left\langle n(q(0)), T_{*}(0)\right)\right\rangle=0$ by previous results, we see that $\left\langle v\left(0^{+}\right), T_{*}(0)\right\rangle=\left\langle v\left(0^{-}\right), T_{*}(0)\right\rangle$, which completes the proof of the claim.

We are now in a position to prove that the billiard in $\tilde{Q}$ has the unidirectionality property. First, note that the invariance of $N$ implies that if $v_{*}(\bar{t})=0$ for some billiard orbit and some $\bar{t} \in \mathbb{R}$, then $v_{*}$ is identically zero along that orbit. Now, suppose that the billiard in $\tilde{Q}$ does not have the unidirectionality property. Then, we can find a billiard orbit such that $v_{*}\left(t_{1}\right) v_{*}\left(t_{2}\right)<0$ for some $t_{1}<t_{2}$. Since $v_{*}$ is continuous, there exists $t_{1}<\bar{t}<t_{2}$ for which $v_{*}(\bar{t})=0$. By previous observation, it follows that $v_{*} \equiv 0$, which contradicts $v_{*}\left(t_{1}\right) v_{*}\left(t_{2}\right)<0$.

We now prove the undirectionality property for billiards in 2-dimensional tracks.

Corollary 1. A billiard in a track $Q \subset \mathbb{R}^{2}$ has the unidirectionality property.

Proof. Embed $Q$ in $\mathbb{R}^{3}$, namely, identify $\mathbb{R}^{2}$ with some plane $P \subset \mathbb{R}^{3}$, and denote by $\tilde{Q}$ the tubular neighborhood of $\gamma$ in $\mathbb{R}^{3}$ with circular section $\Omega$. Clearly, $Q=\tilde{Q} \cap P$. Next, choose the parametrization $\zeta$ of the circle $\partial \Omega$ so that $F(s) \zeta(0) \in P$. In other words, we require the curve $F(s) \zeta(\alpha)$ to intersect $P$ for $\alpha=0$. If $\gamma$ is a straight segment, then in order for the previous statement to make sense, the vector $N(s)$ has to be chosen so to lie on $P$ (recall that $N$ and $B$ are arbitrarily selected if $\gamma$ is a straight segment). Now, let $\tilde{n}$ be as in the proof of Proposition 1 . The corollary will be proved once we show that $\tilde{n}(s, 0)=\lambda N(s)$ for some $\lambda \neq 0$. Indeed, from (1) and the fact that $\tau \equiv 0$, we obtain immediately that $\tilde{n}(s, 0)=-\left(1-\zeta_{1}(0) \kappa(s)\right) N(s)$. To complete the proof, just note that $\left|\zeta_{1}(0) \kappa(s)\right|<1$ by assumption.

2.3. Main result. The map $T$ is called (nonuniformly) hyperbolic if all its Lyapunov exponents are non-zero almost everywhere on $\tilde{M}$.

Definition 1. We say that a circular guide is of type $\mathrm{A}$ if $\alpha \geq \pi$ (and no conditions on $r_{1}$ and $r_{2}$ are imposed), and is of type $\mathrm{B}$ if $r_{2} / r_{1}<1 / 2$ (and no conditions on $\alpha$ are imposed).

We now state the main result of this paper.

Theorem 1. Let $Q$ be a track, and suppose that each circular guide of $Q$ is of type $A$ or $B$. Then the billiard map $T$ in $Q$ is hyperbolic provided that the straight guides of $Q$ are sufficiently long.

To our knowledge, all the recipes for designing hyperbolic billiard domains including focusing and dispersing in their boundaries require these curves to be placed sufficiently apart [B1,C-M,M1,W2,W3]. Since for guides of type A, there is no restriction on the distance between the outer and inner circles, Theorem 1 tells us that there do exist hyperbolic billiard domains that violate the condition on the separation between focusing and 


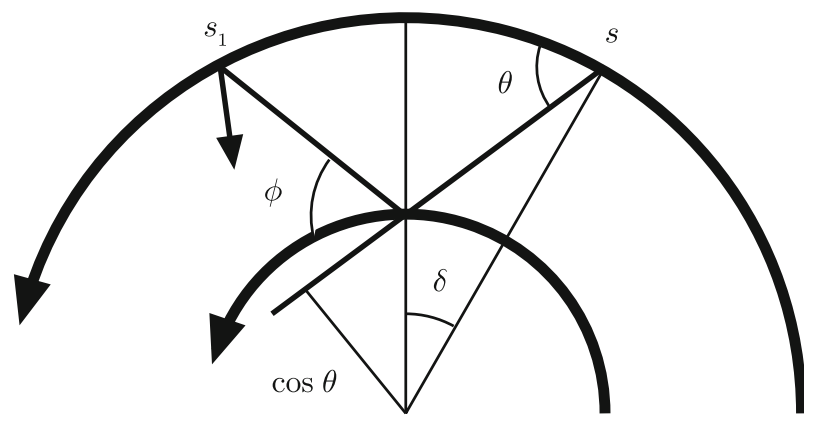

Fig. 3. Consecutive collisions inside a circular guide

dispersing boundary components. This means that the mechanism generating hyperbolicity in these billiards is not the defocusing one, which requires that after a reflection off of a focusing curve, an infinitesimal family of parallel trajectories must focus and defocus before the next collision with the boundary of the billiard table. However, we have to point out that circular guides are very special domains, because the billiards inside them are integrable. Also, note that we still need to put circular guides sufficiently far away from each other in order to obtain hyperbolicity. While writing this paper, we learned that Bussolari and Lenci also constructed hyperbolic billiards (different than track billiards) that violate the aforementioned separation condition [B-L].

2.4. Billiard dynamics in a circular guide. To prove Theorem 1 , it is essential to investigate the billiard dynamics inside a circular guide.

Consider a circular guide with outer and inner radii $r_{1}=1$ and $0<r_{2}=r<1$, respectively. Note that by a proper rescaling, every circular guide can be transformed into such a guide. Denote by $M_{1}$ the set of all collisions $(q, v)$ such that $q$ belongs to the outer circle of the guide. Note that the ray $\{q+t v: t \geq 0\}$ emerging from $x=$ $(q, v) \in M_{1}$ is tangent to the full circle containing the inner arc of the guide if and only if $\theta(x) \in\{\bar{\theta}, \pi-\bar{\theta}\}$, where $\bar{\theta}=\cos ^{-1} r \in(0, \pi / 2)$. Let $D_{1}=M_{1} \backslash \theta^{-1}(\{0, \pi, \bar{\theta}, \pi-\bar{\theta}\})$. We say that a collision $x \in D_{1}$ is 'leaving (the guide)' if the only point of intersection between the ray emerging from $x=(q, v)$ and the guide is $q$. We say that a collision $x \in D_{1}$ is 'entering (the guide)' if $-x$ is leaving the guide. For every $x \in D_{1}$, denote by $n_{1}(x) \geq 0$ the number of times that the particle with initial state $x$ hits the outer circle before leaving the guide.

We will focus our attention on the transformation $T_{1}: D_{1} \rightarrow D_{1}$ that maps a collision with the outer circle to the next collision with the same circle. More precisely, for every $(s, \theta) \in D_{1}$, define

$$
T_{1}(s, \theta)= \begin{cases}(s+2 \delta(\theta), \theta) & \text { if } n_{1}(x)>0 \\ (s, \theta) & \text { if } n_{1}(x)=0\end{cases}
$$

where $2 \delta(\theta)$ is the central angle of the sector bounded by the two consecutive collisions with the outer circle (see Fig. 3). For $\theta \in(0, \bar{\theta}) \cup(\pi-\bar{\theta}, \pi)$, it is trivial to check that $\delta(\theta)=\theta$. For $\theta \in(\bar{\theta}, \pi-\bar{\theta})$ instead, we see from Fig. 3 that $\delta(\theta)=\theta-\phi(\theta)$, where $\phi(\theta)$ is the angle of the collision with the inner circle. The relation between $\theta$ and $\phi$ is provided by the conservation of the angular momentum of the particle measured from 
the center of the circular guide, which reads as $\cos \theta=r \cos \phi$. Putting all together, we obtain

$$
\delta(\theta)= \begin{cases}\theta-\cos ^{-1}\left(\frac{\cos \theta}{r}\right) & \text { if } \theta \in(\bar{\theta}, \pi-\bar{\theta}), \\ \theta & \text { if } \theta \in(0, \bar{\theta}) \cup(\pi-\bar{\theta}, \pi) .\end{cases}
$$

The function $\delta$ is differentiable on $(0, \pi) \backslash\{\bar{\theta}, \pi-\bar{\theta}\}$, and $\delta^{\prime}(\theta) \rightarrow-\infty$, as $\theta \rightarrow \bar{\theta}^{+}$or $\theta \rightarrow(\pi-\bar{\theta})^{-}$. By abuse of notation, we define $\delta(x)=\delta(\theta(x))$ and $\delta^{\prime}(x)=\delta^{\prime}(\theta(x))$ for every $x \in D_{1}$.

From (2), it follows that for every $x \in D_{1}$,

$$
D_{x} T_{1}^{n_{1}(x)}=\left(\begin{array}{cc}
1 & 2 n_{1}(x) \delta^{\prime}(x) \\
0 & 1
\end{array}\right) .
$$

2.5. Preliminary lemmas. We now prove some facts that will play a crucial role in the proof the hyperbolicity of track billiards. The goal here is to estimate the quantity $2 n_{1}(x) \delta^{\prime}(x)$ for $x \in D_{1}$.

Definition 2. Let $E_{1}=\left\{x \in D_{1}: x\right.$ is entering and $T_{1}^{n_{1}(x)} x$ is leaving $\}$. The set $E_{1}$ can be partitioned as follows $E_{1}=E_{0} \cup E_{+} \cup E_{-}$, where

- $E_{0}=\left\{x \in E_{1}: n_{1}(x)=0\right\}$,

- $E_{+}=\left\{x \in E_{1} \backslash E_{0}: \theta(x) \in(\bar{\theta}, \pi-\bar{\theta})\right\}$,

- $E_{-}=\left\{x \in E_{1} \backslash E_{0}: \theta(x) \in(0, \bar{\theta}) \cup(\pi-\bar{\theta}, \pi)\right\}$.

For every $x \in E_{1}$, define

$$
\omega(x)=\alpha-2 n_{1}(x) \delta(x),
$$

and

$$
\chi(x)=2 n_{1}(x) \delta^{\prime}(x) .
$$

Remark 1. From the definition of $\omega(x)$, it follows that $0 \leq \omega(x)<2 \delta(x)$ for every $x \in E_{1}$.

The next lemma is a trivial consequence of the definition of $E_{0}$ and the fact that $\delta^{\prime}(x)=1$ for all $x \in E_{-}$.

Lemma 1. If $x \in E_{0} \cup E_{-}$, then $\chi(x)=2 n_{1}(x)$.

We now restrict our analysis to the circular guides of type A and B.

Lemma 2. Consider a circular guide of type A. There exists a function $\chi_{A}=\chi_{A}(r, \alpha)$ non-increasing in $\alpha$ such that $\chi(x) \leq \chi_{A}$ for every $x \in E_{+}$.

Proof. By the symmetry of the guide, it is enough to prove the lemma for $x \in E_{+}$such that $\theta(x) \in(\bar{\theta}, \pi / 2)$. For such values of $x$, we have

$$
\delta^{\prime}(\theta)=1-\frac{\sin \theta}{\sqrt{r^{2}-\cos ^{2} \theta}}<0
$$


and

$$
\delta^{\prime \prime}(\theta)=\frac{\cos \theta}{\left(r^{2}-\cos ^{2} \theta\right)^{\frac{3}{2}}}\left(1-r^{2}\right)>0 .
$$

Since $\delta^{\prime}(\theta) \rightarrow-\infty$, as $\theta \rightarrow \bar{\theta}^{+}$, we can find $\vartheta \in(\bar{\theta}, \pi / 2)$ such that $\chi(x)<-3$ for every $x \in E_{+}$with $\theta(x) \in(\bar{\theta}, \vartheta]$.

We now consider the case $x \in E_{+}$with $\theta(x) \in(\vartheta, \pi / 2)$. It is trivial to see that for every $\theta \in(\vartheta, \pi / 2)$, we have $\delta(\theta)=-\delta^{\prime}(\theta) \Delta \theta$, where $\Delta \theta$ is the length of the segment lying on the $\theta$-axis whose endpoints are $\theta$ and the intersection point of the tangent of the graph of $\delta$ at $(\theta, \delta(\theta))$ with the $\theta$-axis. Since $\delta$ is strictly convex and $\delta(\pi / 2)=0$, it follows that $0<\Delta \theta<\pi / 2-\theta$ for every $\theta \in(\vartheta, \pi / 2)$. Hence

$$
\frac{\delta^{\prime}(\theta)}{\delta(\theta)}=-\frac{1}{\Delta \theta}<-\frac{2}{\pi-2 \theta} \quad \text { for } \theta \in(\vartheta, \pi / 2) .
$$

Now, note that $\alpha-\omega(x)>0$ because $n_{1}(x)>0$. Combining together the last observation, inequality (5) and Remark 1, we obtain

$$
\begin{aligned}
\chi(x) & =(\alpha-\omega(x)) \frac{\delta^{\prime}(\theta(x))}{\delta(\theta(x))} \\
& <-2 \frac{\alpha-\omega(x)}{\pi-2 \theta(x)} \\
& <-2 \frac{\alpha-2 \delta(\theta(x))}{\pi-2 \theta(x)} \quad \text { for } \theta(x) \in(\vartheta, \pi / 2) .
\end{aligned}
$$

Let $h(\alpha, \theta)=-2(\alpha-2 \delta(\theta)) /(\pi-2 \theta)$. Since $\pi \leq \alpha$ and $\delta(\theta)<\theta$, it is easy to see that $\partial_{\alpha} h<0$ and $h(\alpha, \vartheta)<-2$. So $h$ is strictly decreasing, and therefore

$$
\chi(x)<h(\alpha, \vartheta)<-2 \quad \text { for } \theta(x) \in(\vartheta, \pi / 2) .
$$

To complete the proof, set $\chi_{A}=\max \{-3, h(\alpha, \vartheta)\}$, and observe that $\chi_{A}$ is a nonincreasing function of $\alpha$.

Since $\delta^{\prime}$ is strictly increasing for $\theta \in(\bar{\theta}, \pi / 2)$ (see the Proof of Lemma 2), we have $\delta^{\prime}(x)<\delta^{\prime}(\pi / 2)=1-1 / r$ for every $x \in D_{1}$ such that $\theta(x) \in(\bar{\theta}, \pi-\bar{\theta})$. This simple fact proves immediately the following lemma, saying that a result similar to Lemma 2 holds true for circular guides of type B.

Lemma 3. Consider a guide of type $B$, and let $\chi_{B}=\chi_{B}(r)=2(1-1 / r)<-2$. Then $\chi(x) \leq 2 n_{1}(x)(1-1 / r)<\chi_{B}$ for every $x \in E_{+}$.

Remark 2. It is precisely the fact that $|\chi(x)|>2$, proved in the previous lemmas, which allows us to think of circular guides as optical devices having the property of focusing in a controlled way infinitesimal families of parallel rays entering the guide. In this sense, we can think of circular guides of type A and B as some sort of generalized absolutely focusing curves $[\mathrm{B} 2, \mathrm{D}]$.

\section{Hyperbolicity}

In this section, we prove that, under proper conditions concerning the circular guides and the distance between them, a track billiard admits an eventually strictly invariant cone field. By a well known result of Wojtkowski [W2], this property implies Theorem 1. 
3.1. Focusing times. Recall that $M$ is the phase space of the billiard inside the track $Q$. Given a tangent vector $u \in T_{x} M$ at $x \in$ int $M$, let $s \mapsto \gamma(s)=(q(s), v(s)) \in$ int $M$ be a differentiable curve such that $\gamma(0)=x$ and $\gamma^{\prime}(0)=u$. Next, define a family of lines $s \mapsto \gamma_{+}(s)$ by setting $\gamma_{+}(s)=\{q(s)+t v(s): t \in \mathbb{R}\}$. Similarly, define a second family of lines $s \mapsto \gamma_{-}(s)$ by replacing $\gamma$ with $-\gamma$ in the definition of $\gamma_{+}$. In geometrical terms, $\gamma_{-}$is obtained from $\gamma_{+}$by reflecting its lines at $\partial Q$. All the lines of $\gamma_{+}\left(\gamma_{-}\right)$intersect in linear approximation at one point along the line $\gamma_{+}(0)\left(\gamma_{-}(0)\right)$. This point is called a focal point of $u$. If $x=(s, \theta)$ and $u=(d s, d \theta)$, then the distances between $\pi(x)$ and the focal points of $u$ lying on $\gamma_{+}(0)$ and $\gamma_{-}(0)$ are, respectively, given by

$$
f_{+}(u)=\frac{\sin \theta}{\kappa(s)+m(u)}
$$

and

$$
f_{-}(u)=\frac{\sin \theta}{\kappa(s)-m(u)},
$$

where $\kappa(s)$ is the curvature $\partial Q$ at $s$ and $m(u)=d \theta / d s$ (see for example, [W2]). We conventionally assume that the curvature of the outer circle is positive, whereas the curvature of the inner circle is negative. The distances $f_{+}(u)$ and $f_{-}(u)$ are called forward and backward focusing times of $u$. By summing the reciprocals of $f_{+}(u)$ and $f_{-}(u)$, we obtain the well known Mirror Formula ${ }^{1}$

$$
\frac{1}{f_{+}(u)}+\frac{1}{f_{-}(u)}=\frac{2 \kappa(s)}{\sin \theta} .
$$

\subsection{Fractional linear transformation}

Definition 3. Let $E$ be the set of all collisions $x \in M \backslash S_{1}^{+}$entering a circular guide of $Q$. Also, for every $x \in E$, denote by $n(x) \geq 0$ the times that the particle with initial state $x$ hits the boundary of the circular guide before leaving it.

Following [W3], we now introduce a transformation describing the relation between the focusing times of an infinitesimal family of billiard trajectories at the entrance and at the exit of a circular guide.

Let $x \in E$, and consider $0 \neq u \in T_{x} M$. Next, denote by $F_{x}$ the map from the real projective line $\mathbb{R} \cup\{\infty\}$ to itself given by $f_{-}(u) \mapsto f_{+}\left(D_{x} T^{n(x)} u\right)$. Using the Mirror Formula, one can deduce that $F_{x}$ is a linear fractional transformation (restricted to $\mathbb{R} \cup\{\infty\})$

$$
F_{x}(f)=\frac{a(x) f+b(x)}{c(x) f+d(x)} \quad \text { for } f \in \mathbb{R} \cup\{\infty\},
$$

where $a(x), b(x), c(x), d(x)$ are real numbers satisfying $a(x) d(x)-b(x) c(x)<0$. For $x \in E_{1}$, the analytic expression of $a(x), b(x), c(x), d(x)$ will be derived in the proof of Theorem 2. The inequality $a(x) d(x)-b(x) c(x)<0$ implies that $d F_{x} / d f<0$ is negative on $\mathbb{R}$. Therefore, the transformation $F_{x}$ has two fixed points $f_{1}(x)$ and $f_{2}(x)$ on the real line. We will always assume that $f_{1}(x) \geq f_{2}(x)$. The following lemma is an immediate consequence of the monotonicity of $F_{x}$.

\footnotetext{
1 The convention on the signs of the focusing times adopted here is different than that used in [W2].
} 
Lemma 4. For every $x \in E$, we have

$$
f<f_{2}(x) \text { or } f>f_{1}(x) \Longleftrightarrow f_{2}(x)<F_{x}(f)<f_{1}(x) .
$$

Definition 4. We call the focal length of a circular guide the number

$$
\tilde{f}=\sup _{x \in E} f_{1}(x) .
$$

In the next theorem, we prove that the focal length of a circular guide of type A or B is always bounded above.

Theorem 2. Let $\tilde{\chi}=\chi_{A}$ for a guide of type $A$, and $\tilde{\chi}=\chi_{B}$ for a guide of type $B$. Then

$$
\tilde{f} \leq \frac{\tilde{\chi}}{\tilde{\chi}+2}
$$

Proof. We first prove that $\sup _{x \in E_{1}} f_{1}(x) \leq \tilde{\chi} /(2+\tilde{\chi})$. To this end, we need to compute the fixed point of $F_{x}$ for $x \in E_{1}$. Note that $n(x)=n_{1}(x)$ in this case. By (6) and (7), we have $f=f_{-}(u)=\sin \theta(x)(1-m(u))^{-1}$ and $f_{+}\left(D_{x} T^{n_{1}(x)} u\right)=\sin \theta(x)(1+$ $\left.m\left(D_{x} T^{n_{1}(x)} u\right)\right)^{-1}$ for $0 \neq u \in T_{x} M$. It follows from (4) that $m\left(D_{x} T^{n_{1}(x)} u\right)=m(u)(1+$ $\chi(x) m(u))^{-1}$. A straightforward computation yields

$$
F_{x}(f)=\frac{\sin \theta(x)(1+\chi(x)) f-\sin \theta(x) \chi(x)}{(2+\chi(x)) f-\sin \theta(x)(1+\chi(x))} .
$$

By Lemmas 1, 2 and 3, we know that $\chi(x) \geq 0$ for $x \in E_{0} \cup E_{-}$, and $\chi(x)<-2$ for $x \in E_{+}$. It is then easy to check that the fixed points of $F_{x}$ are given by

$$
f_{1}(x)= \begin{cases}\sin \theta(x) & \text { if } x \in E_{0} \cup E_{-}, \\ \frac{\sin \theta(x) \chi(x)}{2+\chi(x)} & \text { if } x \in E_{+},\end{cases}
$$

and

$$
f_{2}(x)= \begin{cases}\frac{\sin \theta(x) \chi(x)}{2+\chi(x)} & \text { if } x \in E_{0} \cup E_{-}, \\ \sin \theta(x) & \text { if } x \in E_{+} .\end{cases}
$$

Observe that $\chi(x)(2+\chi(x))^{-1} \geq 1$ for $x \in E_{+}$, and the function $z \mapsto z(z+2)^{-1}$ is increasing for $z \in(-\infty,-2)$. Hence

$$
\begin{aligned}
\sup _{x \in E_{1}} f_{1}(x) & \leq \sup _{x \in E_{+}} \frac{\chi(x)}{2+\chi(x)} \\
& \leq \frac{\sup _{x \in E_{+}} \chi(x)}{2+\sup _{x \in E_{+}} \chi(x)} \\
& \leq \frac{\tilde{\chi}}{2+\tilde{\chi}} .
\end{aligned}
$$

We now consider the case $x \in E \backslash E_{1}$. This time, rather than computing directly the fixed points of $F_{x}$, we will try to reduce the current case to the one studied in the first part of this proof. This will be done by considering our guide as contained in a larger guide. In fact, a circular guide can always be embedded into a larger guide: the radii of the outer and inner circles of the larger guide coincide with those of the original guide, 


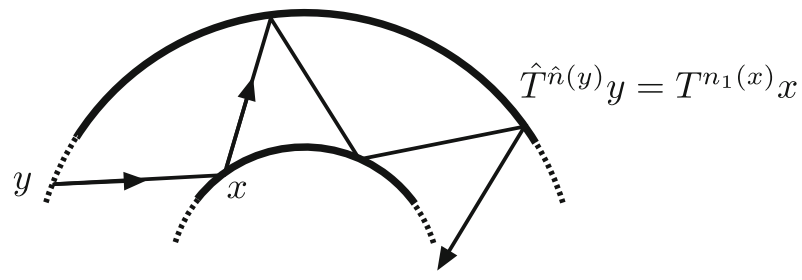

Fig. 4. The solid and dotted curves denote the original and the enlarged guide, respectively, as described in the proof of Theorem 2

but if $\beta$ and $\alpha$ are the central angle of the larger and the original guide, respectively, then $\beta>\alpha$. In the rest of this proof, the symbols denoting the billiard transformation and related mathematical objects for the original guide will be used with the addition of a hat to denote their counterparts for the larger guide. Thus, for example, $\hat{M}$ denotes the set of all possible collisions for the larger guide. Note that the sets $M$ and $\hat{M}$ are subsets of the unit tangent bundle of $\mathbb{R}^{2}$. Accordingly, given $x \in M$ and $y \in \hat{M}$, we write $x=y$ if $x$ and $y$ coincide as tangent vectors of $\mathbb{R}^{2}$.

After this parenthesis on the notation, we can resume our proof. We embed the original guide into a larger guide so that there exists $y \in \hat{E}_{1}$ with $n(x) \leq \hat{n}(y) \leq n(x)+2$ for which $\left\{x, \ldots, T^{n(x)} x\right\} \subset\left\{y, \ldots, \hat{T}^{\hat{n}(y)} y\right\}$. The condition $n(x) \leq \hat{n}(y) \leq n(x)+2$ implies $\hat{T} y=x$ or $\hat{T}^{\hat{n}_{1}(y)-1} y=T^{n(x)} x$. We will study only the case $\hat{T} y=x$, because the case $y=x$ can be studied similarly. The case $\hat{T} y=x$ can be further split into two subcases: i) $\hat{T} y=x$ and $\hat{T}^{\hat{n}_{1}(y)-1} y=T^{n(x)} x$ and ii) $\hat{T} y=x$ and $\hat{T}^{\hat{n}_{1}(y)} y=T^{n(x)} x$. Again, we will only consider the second subcase, because the first can be studied similarly (as a matter of fact, its analysis is easier). Hence, we assume that $\hat{T} y=x$ and $\hat{T}^{\hat{n}_{1}(y)} y=T^{n(x)} x$.

Denote by $f_{+}$the difference between the length of the segment connecting $\pi(x)$ with $\pi(y)$ and $f_{1}(x)$. Next, $f_{-}$can be found by using the Mirror Formula $f_{-}^{-1}+f_{+}^{-1}=$ $2 / \sin \hat{\theta}(y)$. Note that $\hat{\theta}(y)=\theta(x)$. By the definition of the map $F_{x}$, it follows that $\hat{F}_{y}\left(f_{-}\right)=F_{x}\left(f_{1}\right)$ (see Fig. 4$)$, and so

$$
\hat{F}_{y}\left(f_{-}\right)=f_{1}(x)
$$

We want to show that $f_{1}(x) \leq \hat{f}_{1}(y)$. To do this, we argue by contradiction. Suppose that $f_{1}(x)>\hat{f}_{1}(y)$. Since $y \in \hat{E}_{1}$, we know from the first part of this proof that the fixed points of $\hat{F}_{y}$ satisfy $\sin \hat{\theta}(y)=\hat{f}_{2}(y)<\hat{f}_{1}(y)$. Also, since $\hat{\theta}(y) \in(\bar{\theta}, \pi-\bar{\theta})$, it is easy to check that the length of the segment connecting $\pi(x)$ with $\pi(y)$ is less than $\sin \hat{\theta}(y)$. Hence $f_{+}<0$. The Mirror Formula then implies that $0<f_{-}<(\sin \hat{\theta}(y)) / 2<\hat{f}_{2}(y)$. By Lemma 4, it follows that $\hat{f}_{2}(y)<\hat{F}_{y}\left(f_{-}\right)<\hat{f}_{1}(y)$, and so $\hat{f}_{2}(y)<f_{1}(x)<\hat{f}_{1}(y)$ by (9). The last inequality contradicts our assumption. Thus $f_{1}(x) \leq \hat{f}_{1}(y)$.

By the first part of this proof, the right-hand side part of the previous inequality is bounded above by $\tilde{\chi}(\beta)(2+\tilde{\chi}(\beta))^{-1}$. Since $\tilde{\chi}(\beta)$ is non-increasing in $\beta$ (in fact, $\tilde{\chi}$ is independent of $\beta$ for a guide of type B), we conclude that $f_{1}(x) \leq \tilde{\chi}(\alpha)(2+\tilde{\chi}(\alpha))^{-1}$. This completes the proof. 
3.3. Cone fields. A cone in a 2-dimensional space $V$ is a subset

$$
\mathcal{C}=\left\{a X_{1}+b X_{2}: a b \geq 0\right\},
$$

where $X_{1}$ and $X_{2}$ are two linear independent vectors of $V$. Equivalently, we can say that the cone $\mathcal{C}$ is a closed interval of the projective space $\mathbb{P}(V)$, the space of the lines in $V$. The interior of $\mathcal{C}$ is defined by int $\mathcal{C}=\left\{a X_{1}+b X_{2}: a b>0\right\} \cup\{0\}$. Since the backward focusing time $f_{-}$and the forward focusing time $f_{+}$are both projective coordinates of $\mathbb{P}\left(T_{x} M\right)$, the set $\mathcal{C}=\left\{u \in T_{x} M: f_{-}(u)\left(f_{+}(u)\right) \in I\right\}$ is a cone in $T_{x} M$ for every closed interval $I \subset \mathbb{R}$.

Let $\Lambda$ be a subset of $\tilde{M}$ such that $\mu(\Lambda)>0$. Denote by $T_{\Lambda}: \Lambda \rightarrow \Lambda$ the first return map on $\Lambda$ induced by the billiard map $T$. Also, denote by $\mu_{\Lambda}$ the probability measure on $\Lambda$ obtained by normalizing the restriction of $\mu$ to $\Lambda$. It is well known that the map $T_{\Lambda}$ preserves $\mu_{\Lambda}$.

Definition 5. A measurable cone field $\mathcal{C}$ on $\Lambda$ is a measurable map that associates to each $x \in \Lambda$ a cone $\mathcal{C}(x) \subset T_{x} M$. We say that $\mathcal{C}$ is eventually strictly invariant if for every $x \in \Lambda$, we have

1. $D_{x} T_{\Lambda} \mathcal{C}(x) \subset \mathcal{C}\left(T_{\Lambda} x\right)$,

2. $\exists$ an integer $k(x)>0$ such that $D_{x} T_{\Lambda}^{k(x)} \mathcal{C}(x) \subset \operatorname{int} \mathcal{C}\left(T_{\Lambda}^{k(x)} x\right)$.

Remark 3. By [W2], the existence of such a cone field (plus other properties, always satisfied by track billiards) implies that $T_{\Lambda}$ is hyperbolic. Furthermore, if the set $\cup_{k \in \mathbb{Z}} T^{k} \Lambda$ has full $\mu$-measure, then it is not difficult to see that $T$ is hyperbolic as well (see [W1]).

We now define an invariant cone field for circular track billiards. In the next subsection, we will show, relying on Lemmas 2 and 3 and Theorem 2, that this cone field is eventually strictly invariant provided that the straight guides of a track are sufficiently long.

Let $\tilde{E}=E \cap \tilde{M}$ be the set of entering collisions with infinite positive and negative semi-orbits. We define a measurable cone field on $\tilde{E}$ as follows:

$$
\mathcal{C}(x)=\left\{u \in T_{x} M: f_{-}(u) \geq \tilde{f}(x)\right\} \quad \text { for all } x \in \tilde{E},
$$

where $\tilde{f}(x)$ is the focal length of the circular guide containing $\pi(x)$. The cone field $\mathcal{C}$ is continuous on $\tilde{E}$ (and therefore measurable), because so are $f_{-}$(as a function of $x$ ) and $\tilde{f}$.

3.4. Hyperbolicity. Let $Q$ be a track, and assume that its guides are ordered in such a way that the $i^{\text {th }}$ straight guide connects the $i^{\text {th }}$ and $(i+1)^{\text {th }}$ circular guides. The $(n+1)^{\text {th }}$ circular guide coincides with the first one so that there are exactly $n$ circular guides separated by $n$ straight guides. We also assume that each circular guide is of type A or B. For every $1 \leq i \leq n$, let $\tilde{f}_{i}$ and $l_{i}$ be the focal length and the length of the $i^{\text {th }}$ circular guides and the $i^{\text {th }}$ straight guide, respectively. We say that such a track $Q$ satisfies Condition $\mathrm{H}$ if the distance between any pair of consecutive circular guides of $Q$ is greater than the focal length of the two circular guides, i.e.,

$$
l_{i}>\tilde{f}_{i}+\tilde{f}_{i+1} \quad \text { for each } i=1, \ldots, n .
$$

We can now give the precise formulation and the proof of Theorem 1, the main result of this paper. 
Theorem 3. Suppose that a track $Q$ satisfies Condition $H$. Then the billiard map $T$ in $Q$ is hyperbolic.

Proof. By Remark 3, it is enough to prove that the cone field $\mathcal{C}$ defined in (10) is eventually strictly invariant, and the set $\cup_{k \in \mathbb{Z}} T^{k} \tilde{E}$ has full $\mu$-measure.

Let $x \in \tilde{E}$, and consider $u \in \mathcal{C}(x)$ with $u \neq 0$. By definition of $\mathcal{C}(x)$, we have $f_{-}(u)>\tilde{f}(x) \geq f_{1}(x)$ so that Lemma 4 implies that $f_{+}\left(D_{x} T^{n(x)} u\right)<f_{1}(x) \leq \tilde{f}(x)$. Now, note that $T^{n(x)} x$ is a collision leaving a circular guide, and that the piece of the orbit of $x$ between $x$ and $T_{\tilde{E}} x$ crosses a straight guide of length $l$. By Condition $\mathrm{H}$, we then have $l>\tilde{f}(x)+\tilde{f}\left(T_{\tilde{E}} x\right)$, and hence

$$
\begin{aligned}
f_{-}\left(D_{x} T_{\tilde{E}} u\right) & =l-f_{+}\left(D_{x} T^{n(x)} u\right) \\
& \geq l-\tilde{f}(x) \\
& >\tilde{f}\left(T_{\tilde{E}} x\right) .
\end{aligned}
$$

This means that $D_{x} T_{\tilde{E}} u \in \operatorname{int} \mathcal{C}\left(T_{\tilde{E}^{x}}\right)$, and we can conclude that $\mathcal{C}$ is eventually strictly invariant with $k(x)=1$ for every $x \in \tilde{E}$. It is clear that $\cup_{k \in \mathbb{Z}} T^{k} \tilde{E}=\tilde{M} \backslash N$ (for the definition of $N$, see Subsect. 2.2). Since $\mu(N)=0$, it follows that $\cup_{k \in \mathbb{Z}} T^{k} \tilde{E}$ has full measure.

Remark 4. It is easy to check that the so-called Monza billiard considered in [V-P-R] satisfies Condition H. Note that its circular guides are of type B. Theorem 3 then assures that the Monza billiard is hyperbolic.

\section{3-Dimensional Track Billiards}

In this last section, we extend Theorem 3 to billiards in 3-dimensional tracks, which are special 3-dimensional tubular domains with rectangular cross section.

A 3-dimensional cylindrical (straight) guide $\tilde{G}$ is the Cartesian product of a 2-dimensional circular (straight) guide $G$ and a closed interval $I \subset \mathbb{R}$. The guide $G$, when circular, is assumed to be of type A or B. We call the axis and the focal length of $\tilde{G}$ the line perpendicular to the plane containing $G$ and focal length of $G$, respectively. The two rectangles of $\partial \tilde{G}$, each being the Cartesian product of an opening of $G$ and $I$, are called the ends of $\tilde{G}$.

We say that a cylindrical guide $\tilde{G}_{1}$ and a straight guide $\tilde{G}_{2}$ are glued together if there exists an isometry of $\mathbb{R}^{3}$ that identifies one end of $\tilde{G}_{1}$ with one end of $\tilde{G}_{2}$.

Definition 6. A3-dimensional track is a finite chain of alternating 3-dimensional straight and cylindrical guides glued together. More precisely, a connected subset $\tilde{Q} \subset \mathbb{R}^{3}$ is called a 3-dimensional track if $\tilde{Q}$ is a union of 3-dimensional guides $\tilde{G}_{1}, \ldots, \tilde{G}_{2 n+1}$ with $n>1$ such that

1. $\tilde{G}_{2 n+1}=\tilde{G}_{1}$,

2. $\tilde{G}_{2 i-1}$ and $\tilde{G}_{2 i}$ are a cylindrical and a straight guide, respectively, for each $i=1, \ldots, n$,

3. $\tilde{G}_{i}$ and $\tilde{G}_{i+1}$ are glued together for each $i=1, \ldots, 2 n$.

Note that $\tilde{Q}$ must contain at least two cylindrical guides. An example of a 3-dimensional track is depicted in Fig. 5. 


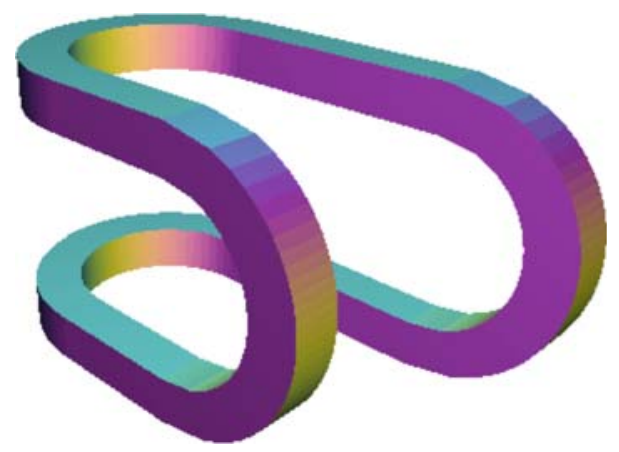

Fig. 5. A 3-dimensional track that satisfies Condition $\tilde{\mathrm{H}}$

Remark 5. A 3-dimensional track is a tubular neighborhood of a curve that is a union of finitely many planar curves (straight segments and arcs of circles) and with rectangular cross section (see Subsect. 2.2). From Proposition 1, it follows that 3-dimensional track billiards have the unidirectionality property.

If a 3-dimensional track has the property that the axes of the cylindrical guides are all parallel to each other, then the momentum of the particle along this line is a first integral of motion, and the billiard is not completely hyperbolic. A billiard inside a 2-dimensional track is hyperbolic if it satisfies Condition $\mathrm{H}$. We now introduce the 3-dimensional analogue of Condition $\mathrm{H}$.

A 3-dimensional track $\tilde{Q}=\cup_{1 \leq i \leq 2 n+1} \tilde{G}_{i}$ satisfies Condition $\tilde{\mathrm{H}}$ if

1. the distance between the ends of each straight guide $\tilde{G}_{2 i}$ is greater than the sum of the focal lengths of the cylindrical guides $\tilde{G}_{2 i-1}$ and $\tilde{G}_{2 i+1}$ for each $i=1, \ldots, n$;

2. there are at least two cylindrical guides with orthogonal axes.

An example of track satisfying $\tilde{H}$ is shown in Fig. 5. Billiards in tracks satisfying Condition $\tilde{\mathrm{H}}$ are closely related to certain hyperbolic semi-focusing cylindrical billiards [B-D1,B-D2], and are examples of twisted Cartesian products [W3]. Theorem 3 combined with the results of [B-D1] (or Theorem 17 of [W3]) implies that for a 3-dimensional track billiard satisfying Condition $\tilde{\mathrm{H}}$, there exists an invariant cone field that is strictly invariant along every orbit connecting two cylindrical guides with orthogonal axes, thus proving the following theorem.

Theorem 4. If a 3-dimensional track $\tilde{Q}$ satisfies Condition $\tilde{H}$, then the billiard map in $\tilde{Q}$ is hyperbolic.

Open Access This article is distributed under the terms of the Creative Commons Attribution Noncommercial License which permits any noncommercial use, distribution, and reproduction in any medium, provided the original author(s) and source are credited.

\section{References}

[B1] Bunimovich, L.: A Theorem on Ergodicity of Two-Dimensional Hyperbolic Billiards. Commun. Math. Phys. 130, 599-621 (1990)

[B2] Bunimovich, L.: On absolutely focusing mirrors. In: Ergodic theory and related topics, III (Güstrow, 1990), Lect. Notes Math. 1514, Berlin-Heidelberg-New York: Springer-Verlag 1992, pp. 62-82 
[B3] Bunimovich, L.: Mushrooms and other billiards with divided phase space, Chaos 11 (2001), 802-808

[B-D1] Bunimovich, L., Del Magno, G.: Semi-focusing billiards: hyperbolicity. Commun. Math. Phys. 262, 17-32 (2006)

[B-D2] Bunimovich, L., Del Magno, G.: Semi-focusing billiards: ergodicity. Erg. Th. Dynam. Sys. 28, 1377-1417 (2008)

[B-L] Bussolari, L., Lenci, M.: Hyperbolic billiards with nearly flat focusing boundaries. Physica D 237, 2272-2281 (2008)

[C-M] Chernov, N., Markarian, R.: Chaotic billiards. Mathematical Surveys and Monographs 127, Providence, RI: Amer. Math. Soc. 2006

[C-F-S] Cornfeld, I., Fomin, S., Sinai, Ya.: Ergodic theory. New York: Springer-Verlag, 1982

[C-D-F-K] Chenaud, B., Duclos, P., Freitas, P., Krejčiřk, D.: Geometrically induced discrete spectrum in circular tubes. Diff. Geom. Appl. 23, 95-105 (2005)

[D] Donnay, V.: Using integrability to produce chaos: billiards with positive entropy. Commun. Math. Phys. 141, 225-257 (1991)

[E-S] Exner, P., Šeba, P.: Bound states in curved quantum waveguides. J. Math. Phys. 30, 2574-2580 (1989)

[G-J] Goldstone, J., Jaffe, R.L.: Bound states in twisting tubes. Phys. Rev. B 45, 14100-14107 (1992)

[H-P] Horvat, M., Prosen, T.: Uni-directional transport properties of a serpent billiard. J. Phys. A: Math. Gen. 37, 3133-3145 (2004)

[K-S] Katok, A., Strelcyn, J.-M.: Invariant manifolds, entropy and billiards; smooth maps with singularities. Lect. Notes Math. 1222, New York: Springer, 1986

[K1] Klingerberg, W.: A course in differential geometry. Graduate Texts in Mathematics 51, New York: Springer-Verlag, 1978

[M1] Markarian, R.: Non-uniformly hyperbolic billiards. Ann. Fac. Sci. Toulouse Math. 6(3), 223-257 (1994)

[P] Peirone, R.: Billiards in Tubular Neighborhoods of Manifolds of Codimension 1. Commun. Math. Phys. 207, 67-80 (1999)

[V-P-R] Veble, G., Prosen, T., Robnik, M.: Expanded boundary integral method and chaotic time-reversal doublets in quantum billiards. New J. Phys. 9, 15 (2007)

[W1] Wojtkowski, M.: Invariant families of cones and Lyapunov exponents. Erg. Th. Dynam. Syst. 5, 145-161 (1985)

[W2] Wojtkowski, M.: Principles for the design of billiards with nonvanishing Lyapunov exponents. Commun. Math. Phys. 105, 391-414 (1986)

[W3] Wojtkowski, M.: Design of hyperbolic billiards. Commun. Math. Phys. 273, 283-304 (2007)

Communicated by G. Gallavotti 